\title{
OFERTA PÚBLICA DE AQUISIÇÕES DE AÇÕES: EVIDÊNCIAS DE VARIAÇÃO DE VALOR NO MERCADO FINANCEIRO
}

\author{
PUBLIC OFFER FOR SHARES ACQUISITIONS: EVIDENCE OF VALUE CHANGE \\ IN THE FINANCIAL MARKET
}

\section{Recebimento: 3/3/2017- Aceite: 11/11/2016- Publicação: 18/04/2017 \\ Processo de Avaliação: Double Blind Review}

\author{
Daniel Jose Machado ${ }^{1}$ \\ Doutorando em Administração \\ Universidade Presbiteriana Mackenzie - CCSA \\ Pontifícia Universidade católica de São Paulo \\ phd.djmachado@gmail.com
}

\section{José Renato Lamberti}

Doutorando em Administração

jrenatojardim@gmail.com

\section{Helene Rebelo}

Doutorando em Administração

Universidade Presbiteriana Mackenzie - CCSA

helenearebelo@gmail.com

\section{RESUMO}

A hipótese de eficiência do mercado em sua forma semiforte, alvitra que os preços se ajustam instantaneamente refletindo a divulgação pública de quaisquer informações relevantes, coibindo oportunidades de retornos anormais arbitrários. $\mathrm{O}$ objetivo deste estudo é verificar a ocorrência de variação no preço das ações, por ocasião da Oferta Pública de Aquisição de Ações (OPA), no mercado acionário brasileiro, no período entre 2006 a 2015. A metodologia da pesquisa utilizada é o estudo de eventos para avaliar possibilidade de retornos anormais de ativos em relação ao mercado. Esta pesquisa se distingue pela quantidade de OPAs (65) ocorridas em um longo período (10 anos) estudados, contribuindo pela agregação, ao aproximar-se da curva normal. Foram testadas as variações no preço das ações no período que antecedeu, durante e imediatamente após o evento. Os resultados encontrados, em relação ao evento de OPA, evidenciam que, em sua maioria, há significância estatística de variação no preço destas ações. A conclusão é que há evidências da hipótese de eficiência do mercado brasileiro em sua forma semiforte, no período estudado, ocasionada pelo evento das OPAs.

\footnotetext{
1 Autor para correspondência: Universidade Presbiteriana Mackenzie. ua da Consolação, 930 - Prédio 45, térreo, Coordenação PPGA - Consolação, São Paulo - SP, Brasil, 01302-907
} 
Palavras-chaves: oferta Pública de Aquisição de Ações (OPA) - Estudo de eventos Mercado eficiente.

\begin{abstract}
The market efficiency hypothesis in its semi-strong form, recommends the establishment of prices adjust instantly reflecting the public disclosure of all relevant information, restricting the opportunities for arbitrary abnormal returns. The aim of this study is to verify the occurrence of the variation of the stock price during the fixed-price self-tender offer, the Brazilian stock market between 2006 and 2015. The methodology used is the event study to assess the possibility of active abnormal returns relative to financial market. This research is distinguished by the number of takeover bids (65) occurred over a long period (10 years) studied, contributing through aggregation, to approximate the normal curve. Changes in the stock price were tested in the sprint, during and immediately after the event. The results were compared to fixed-price self-tender offer event, show that in most cases, is statistically significant change in the price of these parts. The conclusion is that there is evidence of the efficient market hypothesis in its semi-strong form during the study period, caused by the case of takeover bids, the Brazilian stock market.
\end{abstract}

Keywords: Fixed-price self-tender offer; Event study; Efficient market.

\title{
INTRODUÇÃO
}

Do ponto de vista da teoria de finanças, existem três formas de remunerar os acionistas de uma empresa: pagamento de dividendos, recompra de ações e no Brasil, uma opção para o pagamento de dividendos na forma de juros sobre o capital próprio, conforme Etchebest, Costa, Silva, \& Pasqualeto (2014). No pagamento de dividendos a empresa é tributada, enquanto que na forma de juros sobre o capital próprio, a tributação recai sobre o acionista. Entende-se por recompra de ações o mecanismo que inicia quando uma empresa anuncia para o mercado acionário sua intenção em adquirir uma determinada quantidade de ações de sua própria emissão, como esclarecem Berkovitch \& Narayanna (1993) e Gabrielli $\&$ Saito (2003). A partir de então, a empresa encontra-se apta a comprar, bem como qualquer outro investidor, as ações de sua própria emissão na Bolsa de Valores, até uma determinada quantidade máxima e durante um determinado prazo, estipulados por lei.

Existem quatro motivos principais pelos quais as empresas recompram suas próprias ações. O primeiro é obter uma ótima estrutura de capital, por meio de ajustes entre o capital próprio e o capital de terceiros. O segundo motivo é para reduzir o excesso de fluxos de caixa, evitando assim o conflito de agência. O terceiro é utilizar-se do mecanismo de 
recompra de ações como um substituto mais flexível e com vantagens fiscais sobre o pagamento de dividendos, existente na maioria dos mercados mundiais, porém inexistente no mercado brasileiro, devido à desvantagem fiscal. O quarto consiste em uma maneira que determinada companhia usa, para anunciar ao mercado acionário, que suas ações se encontram subavaliadas, após grandes quedas nesse mesmo mercado como um todo, conforme colocado por Ikenberry, Lakonishok \& Vermaelen (1995), Lambrecht \& Myers (2007), Cremers, Nair \& John (2009) e Etchebest, Costa, Silva, \& Pasqualeto (2014). Desses motivos decorrem as questões: a ocorrência de uma Oferta Pública de Aquisição de Ações (OPA) no mercado brasileiro influencia o preço da respectiva ação durante o período do evento? A magnitude da variação de preço, durante o período do evento, corrobora com a hipótese de mercado eficiente na forma semiforte?

O objetivo desta pesquisa é verificar a ocorrência de variação no preço das ações, por ocasião da Oferta Pública de Aquisição de Ações (OPA), no mercado acionário brasileiro, no período entre 2006 a 2015 e avaliar a hipótese de mercado eficiente na sua forma semiforte proposta por Fama (1970).

Esta pesquisa é relevante não apenas pelo número de empresas de capital aberto brasileiras (59) que realizaram o processo de OPA (65) no mercado acionário brasileiro em um período de 10 anos, compreendido entre 2006 e 2015, mas pelo montante expressivo e retorno anormal proporcionado aos acionistas. Este estudo se distingue pela quantidade significativa de eventos analisados, contribuindo pela agregação, para que o comportamento das amostras se aproxime da normalidade.

\section{REVISÃO DA LITERATURA}

Existem três métodos utilizados pelas empresas para recomprar ações, o preço variável com prêmio (dutch-Auction offer), a recompra a preço fixo com prêmio (fixed-price self-tender offer) e a recompra de ações a mercado aberto (open-market share repurchases), sendo que apenas os dois últimos são praticados no Brasil. No open-market share repurchases, a própria companhia emissora adquire as ações, de tal modo que a empresa recompra as ações no mercado acionário como qualquer outro investidor, embora existam restrições legais tanto na quantidade diária quanto no total de ações que a companhia pode recomprar. No fixed-price self-tender offer, a empresa define o número de ações que 
pretende recomprar, bem como a data de expiração e o preço que pretende pagar, normalmente superior ao do mercado, ficando a cargo do acionista vender ou não suas ações, conforme proposto por Lakonishok \& Vermaelen (1990), Comment \& Jarrell (1991), Berkovitch \& Narayanna (1993), Gabrielli \& Saito (2003), Cremers, Nair \& John (2009), Nossa, Lopes \& Teixeira (2010) e Etchebest, Costa, Silva, \& Pasqualeto (2014).

No Brasil o fixed-price self-tender offer é conhecido como Oferta Pública de Aquisição de Ações (OPA), sendo uma oferta irrevogável e irretratável de compra ou permuta de ações e tem como destinatários todos os titulares de ações da mesma espécie e/ou classe, esclarecem Gabrielli \& Saito (2003). No entanto, a OPA não tem sido utilizada no Brasil somente para recompra parcial de ações, é empregada também no fechamento do capital da empresa, seguindo três modalidades, de acordo com Nossa, Lopes \& Teixeira (2010): a) por alienação de controle acionário, b) por cancelamento de registro na CVM (Comissão de Valores Mobiliários) e c) pelo aumento de participação do acionista controlador.

No Brasil, a partir da promulgação das Leis n. 6385 (1976) e n. 6404 (1976), os programas de recompra de ações foram permitidos. Com o tempo, a popularização do mercado de capitais e a publicação da instrução CVM n. 299 (1999), os programas de recompra começaram a chamar mais atenção no que diz respeito à quantidade de eventos anunciados e ao montante transacionado. A Instrução CVM n. 361 (2002) foi atualizada pelas Instruções CVM n. 436 (2006), CVM n. 480 (2009) e CVM n. 487 (2010), que tratam das regras e procedimentos das Ofertas Públicas de Aquisição de Ações (OPA).

Os leilões de OPA geralmente são concluídos no prazo de um mês a partir da data que a operação inicia no mercado, no entanto vários meses ou mesmo anos podem se passar desde a data de anúncio inicial (Dann, 1981). Após o término do prazo estipulado, durante o qual a companhia poderia recomprar suas ações, poderá haver uma nova reunião de conselho administrativo que poderá deliberar, dentre outras coisas, pela prorrogação do prazo para recompra das ações, bem como seu novo programa, subsequente ao primeiro e sobre o destino das ações efetivamente recompradas (Gabrielli \& Saito, 2003). Desta forma, a data de anúncio inicial é definida como sendo o dia de negociação antes do aparecimento do edital de oferta na edição (Dann, 1981). 
Na literatura acadêmica, a recompra de ações vem recebendo bastante atenção devido ao retorno anormal proporcionado aos acionistas, como averiguaram Dann (1981), Vermaelen (1981), Lakonishok \& Vermaelen (1990), Comment \& Jarrell (1991), Ikenberry, Lakonishok \& Vermaelen (1995), Moreira (2000), Gordon (2001), Gabrielli \& Saito (2003), Nossa, Lopes \& Teixeira (2010) e Etchebest, Costa, Silva, \& Pasqualeto (2014).

Pesquisas recentes realizadas no Brasil objetivam quantificar o retorno anormal (AR) no valor das ações em períodos relativos ao processo de OPA:

\begin{tabular}{|c|c|c|c|}
\hline Autores (ano) & $\begin{array}{l}\text { Existência de } \\
\text { AR }\end{array}$ & Variação & Momento \\
\hline Moreira (2000) & Sim & Positiva & Após anúncio da OPA \\
\hline Gordon (2001) & Sim & Positiva & Após anúncio da OPA \\
\hline \multirow{2}{*}{ Gabrielli \& Saito (2003) } & Sim & Negativa & $\begin{array}{l}\text { 1994-1999 (após anúncio da OPA antes } \\
\text { CVM n. 299*) }\end{array}$ \\
\hline & Sim & Positiva & $\begin{array}{c}\text { 2000-2002 (após anuncio da OPA após } \\
\text { CVM n. 299*) }\end{array}$ \\
\hline \multirow{3}{*}{$\begin{array}{l}\text { Nossa, Lopes \& Teixeira } \\
\text { (2010) }\end{array}$} & Sim & Negativa & $\begin{array}{c}\text { 1994-1999 (após anúncio OPA empresas } \\
\text { losers) }\end{array}$ \\
\hline & Não & Nula & $\begin{array}{c}\text { 1994-1999 (após anúncio OPA empresas } \\
\text { winners) }\end{array}$ \\
\hline & $\operatorname{Sim}$ & Positiva & $\begin{array}{c}\text { 2000-2006 (após anúncio da OPA loser and } \\
\text { winner) }\end{array}$ \\
\hline Etchebest et. al. (2014) & Sim & Negativa & Longo prazo (90, 180 e 360 dias $)$ \\
\hline Rogers \& Mamede (2015) & Sim & Positiva & 2004-2013 (após formador de mercado RI) \\
\hline
\end{tabular}

Nota: *Instrução CVM n. 299 (1999).

Quadro 1 - Estudos de eventos sobre OPA. Fonte: Elaborado pelos autores.

Conforme apresentado no Quadro 1, a grande maioria das pesquisas realizadas sobre processos de OPA no mercado acionário brasileiro busca verificar se há retorno anormal no valor das ações envolvidas nessa estratégia de investimento. As pesquisas de Moreira (2000) e Gordon (2001) demonstram retorno anormal positivo após o anúncio de recompra, e os estudos de Gabrielli \& Saito (2003) testam o efeito da regulamentação sobre a recompra de ações, mais especificamente o impacto da instrução da Comissão de Valores Mobiliários (CVM) n. 299 (1999), que estabeleceu novas regras para o mercado acionário. Além do trabalho realizado por Nossa, Lopes \& Teixeira (2010) que verificou se existe o retorno anormal com o anúncio de recompra de ações em empresas vencedoras e perdedoras (winners and losers). 


\subsection{HIPÓTESE DE MERCADO EFICIENTE}

A base da hipótese da eficiência de mercado está na afirmativa de que o preço de um ativo reflete as informações disponíveis sobre a instituição emissora, impossibilitando aos investidores qualquer ganho anormal (retornos superiores ao retorno ajustado ao risco de determinado ativo) (Fama, 1970). O preço deste ativo seria afetado mais lento ou mais rapidamente pelo conteúdo informacional.

Fama (1970) propôs três formas de eficiência de mercado, a primeira delas mostra que os preços refletem toda a informação contida no registro dos preços passados, sendo chamada forma fraca de eficiência, cujo teste consiste em mensurar quão bem o desempenho passado prediz retornos futuros. Na segunda forma, a semiforte, os preços refletem não só o seu comportamento passado, como também o restante da informação publicada, tais como notícias específicas e anúncios sobre distribuição de lucros e dividendos, os testes procuram especificar quão rápido os preços dos ativos refletem as informações públicas (Fama, 1970). Por fim, há a eficiência na forma forte, na qual os preços refletem as informações públicas e as que podem ser obtidas, como as informações privilegiadas, para testá-la, detecta-se algum investidor que possui alguma informação privilegiada, que não está totalmente refletida nos preços (Fama, 1970).

Para verificação da eficiência dos mercados, Fama (1970) enumerou três condições: a) inexistência de custos de transação, b) toda a informação está disponível a custo zero a todos os participantes do mercado e c) todos concordam quanto aos efeitos das informações nos preços atuais dos ativos, assim como em suas distribuições futuras (expectativas homogêneas). Essas condições seriam suficientes, mas não necessárias para a eficiência dos mercados. Por exemplo, até mesmo elevados custos de transação não implicam que, quando a transação ocorra, os preços não reflitam totalmente a informação disponível.

Em estudo posterior, Fama (1991) propôs alterações na denominação das formas de eficiência de mercado, em vez de testar a forma fraca, que mostra apenas o poder dos retornos passados, sugeriu testes mais abrangentes, tratando da previsibilidade de retornos passados, incluindo variáveis como dividendos anuais e taxas de juros. A segunda categoria, de ajuste de preços diante de anúncios públicos, teve seu título mudado para estudo de evento e a terceira categoria, testes da forma forte, teve seu nome alterado para teste de informações privadas, título considerado mais descritível (Fama, 1991). 
Apesar da proposição das alterações das formas de eficiência de mercado (Fama, 1991), este trabalho adota a terminologia anterior, em que a segunda categoria de ajuste de preços diante de anúncios públicos, é denominada forma semiforte de eficiência do mercado (Fama, 1970), pelo uso generalizado na maioria das publicações e forte apelo intuitivo.

A segunda categoria de denominação das formas de eficiência de mercado justifica o emprego do estudo de evento em pesquisas que envolvem processos de OPA, uma vez que podem ocorrer variações nos preços das ações decorrentes de anúncios públicos como se pode observar em Gabrielli \& Saito (2003), Kang, Kim, Liu, Sougho \& Sangho (2006) e Etchebest, Costa, Silva, \& Pasqualeto (2014).

\subsection{REFERENCIAL EMPÍRICO}

Existem retornos anormais positivos para o preço das ações em torno de um anúncio de recompra, de acordo com Cremers, Nair \& John (2009). Com resultados semelhantes em seus trabalhos, Dann (1981), Vermaelen (1981) e Lakonishok \& Vermaelen (1990), encontraram uma média de retorno de $9 \%$ a mais sobre o preço das ações na semana de vencimento da recompra, uma vez que seus testes evidenciam recompras realizadas por intermédio de recompra a preço fixo com prêmio fixed-price self-tender offer, sendo que Dann (1981), obteve OPA a 22,46\% superior, em média, do que o preço de mercado de fechamento no dia anterior ao anúncio da oferta.

Estes fatos ocorrem porque a recompra de ações quando realizada com quantidade e prazo definidos, possui relação positiva com o retorno anormal, especialmente em empresas menores, dando suporte à hipótese da sinalização, observam Vermaelen (1981) e Cremers, Nair \& John (2009). Sobretudo, Nossa, Lopes \& Teixeira (2010) apontam que após o anúncio do programa de recompra de ações, empresas menores atingem retornos superiores aos das empresas maiores. No entanto, Comment \& Jarrell (1991), alertam que recompras anunciadas, durante as nove semanas seguintes ao anúncio da aquisição, estão associadas aos retornos positivos que não são significativamente diferentes do retorno médio fora deste período.

Por outro lado, Comment \& Jarrell (1991) e Ikenberry, Lakonishok \& Vermaelen (1995) sugerem uma correlação negativa entre o anúncio de um programa de recompra de ações e o desempenho do valor da ação da empresa no período anterior ao anúncio. No 
mercado canadense Ikenberry, Lakonishok \& Vermaelen (1995) expõem que as empresas que recompram ações um ano antes do anúncio apresentam um retorno anormal negativo de $-0,35 \%$ ao mês. Já Cremers, Nair \& John (2009) verificaram se retornos anormais diminuem quando o modelo de precificação de ativos é incorporado ao fator aquisição e observaram uma baixa correlação com as carteiras.

Cabe notar que diversas pesquisas apontam retorno anormal no valor das ações após o anúncio da intenção de recompra, como apontam Etchebest, Costa, Silva, \& Pasqualeto (2014). Algumas pesquisas constataram retornos positivos para os períodos subsequentes, enquanto que outras, retornos anormais negativos para os períodos que antecederam o evento, conforme se segue.

Com o objetivo de avaliar os efeitos da OPA no valor do título, Dann (1981) usou uma janela de estimação de $(-10$ e +10$)$ dias úteis analisando retornos anormais de ações no período compreendido entre 1962-1976. Concluiu que existe retornos anormais positivos e evidências que os valores das empresas aumentam. Analisar o comportamento de preços em decorrência da OPA é o objetivo de Vermaelen (1981) e para tanto utilizou uma janela de estimação de $(-10,-60,+10$ e +60$)$ dias úteis no período entre 1962-1977 e concluiu que os retornos anormais positivos são evidências que os valores das empresas aumentam.

Comparar OPA (Fixed-Price Self-Tender Offers), com o leilão de ofertas holandesa (Dutch-Auction Offer) e Mercado aberto de recompra de ações (Open Market Share Repurchases) é o objetivo da pesquisa de Comment \& Jarrell (1991), usando janelas de estimação de $(-10,-20,-30,-40,-50,+10,+20,+30,+40$ e +50$)$ dias úteis no período entre 1995-1988. Também concluíram que os retornos anormais positivos, evidenciam que os valores das empresas aumentam.

Examinar o desempenho das empresas após anúncio de recompra de ações é o propósito do trabalho de Ikenberry, Lakonishok \& Vermaelen (1995). Utilizaram janelas de estimação de $(-10,-20,-250$ e +20$)$ dias úteis e $(+36$ e +48$)$ meses no período compreendido por 1980-1990. Assim como Dann (1981), Vermaelen (1981) e Comment \& Jarrell (1991), os autores também concluíram que os retornos anormais positivos, evidenciam que os valores das empresas aumentam.

Rever a legislação brasileira sobre OPA e impacto sobre acionistas minoritários é a questão abordada por Gabrielli \& Saito (2003) em seus estudos sobre a regulamentação e 
proteção dos minoritários em recompra de ações. Usaram janelas de estimação de (-10 e +65) dias úteis no período de 1994-2002. Concluíram que houveram retornos anormais positivos, com benefícios aos acionistas minoritários em decorrência das normas da CVM.

Comparar o que impulsiona a aquisição estrangeira e interna nos Estados Unidos é objeto de estudo de Kang, Kim, Liu, Sougho \& Sangho (2006), utilizando janelas de estimação de $(-20$ e +20) dias úteis no período de 1980-1998. Concluíram que vendas e demissões são as fontes menos importantes para aquisições estrangeiras do que para as internas, aquisições são semelhante a tomada de poder (takeovers), sinergia é o principal motivo para ocorrência de aquisições estrangeiras.

Verificar se as anomalias de recompra desaparecem ao longo do tempo é o objetivo da pesquisa de Peyer \& Vermaelen (2009). Utilizaram janelas de estimação de (-6, -3, -1 e $+1,+2,+3, \ldots,+48)$ meses no período de 1992-2002. Concluíram que a oportunidade de arbitragem persiste porque o mercado estabelece preços como se o investidor fosse médio, não como o investidor marginal que determina o preço das ações.

O objetivo do estudo de Etchebest, Costa, Silva, \& Pasqualeto (2014) é investigar o cenário de divulgação de OPA no mercado brasileiro. Utilizaram janelas de estimação de ($20,-10,+30,+60,+90,+180$ e +360$)$ dias úteis no período de 2008-2012 e também concluíram que os retornos anormais positivos evidenciam que os valores das empresas aumentam, assim como Dann (1981), Vermaelen (1981) Comment \& Jarrell (1991) e Ikenberry, Lakonishok \& Vermaelen (1995).

Por outro lado, a pesquisa de Rogers \& Mamede (2015), cujo objetivo é analisar a influência do formador de mercado de ações brasileiras, considerando o Fato Relevante (FR) a data de início da função do formador, para a tentativa de encontrar padrões de retornos anormais. Utilizaram janelas de eventos de $(-15$ e +15$)$ dias úteis no período de 2004-2013. Concluíram que retornos anormais positivos existia antes e depois das datas do evento, que pode contradizer a hipótese do mercado eficiente em sua forma semiforte.

Examinar mudanças intertemporais em diversas variáveis de eficiência de preços de eventos de empresas e o impacto dessas variáveis sobre o desaparecimento de retornos anormais em longo prazo é o objeto de estudo de Fu \& Huang (2015). Usaram janelas de estimação de longo prazo (antes de 2002 e depois 2002) e concluíram que o estudo desafia 
a persistência de retornos anormais em longo prazo e qualquer ponto de vista estático na eficiência do mercado.

Com o objetivo de verificar se fora dos EUA recompras estão associadas à menores retornos no anúncio e maiores retornos em excesso de longo prazo, e também examinar as consequências de recompra de ações de mercado aberto em um ambiente global, utilizando mais de 20.000 anúncios de 32 países, Manconi, Peyer \& Vermaelen (2015) usaram janelas de estimação de $(-1$ e $+1,-2$ e +2 e -3 e +3$)$. A conclusão é que os acionistas estão em melhor situação quando a recompra exige apenas a aprovação do conselho, ao invés de autorização dos acionistas, o que sugere que a insistência dos reguladores europeus sobre a aprovação dos acionistas como um instrumento de proteção dos acionistas de recompras é equivocada.

Desenvolver um índice de subvalorização avançado que melhora os retornos anormais em relação ao índice desenvolvido por Peyer \& Vermaelen (2009) foi o desafio de Evgeniou, Fortuny, Nassuphis \& Vermaelen (2016). Para o seu estudo, utilizaram janelas de estimação de $(-6,+12,+24,+36$ e +48) meses no período de 1985-2014. Concluíram que a anomalia da primeira recompra relatada por Ikenberry, Lakonishok e Vermaelen (1995) ainda está viva e robusta. Retornos excedentes de longa duração são grandes, altamente estatisticamente significante e robusto.

Finalmente, Cziraki, Lyandres \& Michaely (2016) examinaram informações contidas na comercialização e anúncios desses eventos, antes da recompra de ações e anúncios de oferta periódica de ações (SEO), através de amostra global de mais de 4.300 recompras e cerca de 1.800 anúncios de SEO. Usaram janelas de estimação de $(-6,-1,+3,+6$ e +12) meses no período de 1986-2011 e concluíram que os resultados sugerem que existe complementaridade entre as informações em anúncios de eventos e que no pré-evento de informações privilegiadas e que as negociações privilegiadas parecem ser especialmente informativas antes de eventos corporativos importantes.

\section{METODOLOGIA E ANÁLISE DE RESULTADOS}

A metodologia de estudo de evento pode avaliar a reação de mercado próxima à data do evento e informar o valor da operação percebido pelos investidores, a métrica é uma medida imediata com base no valor de mercado. Porém, a reação de curto prazo depende também de outros aspectos, tais como probabilidade de ocorrência da operação percebida 
pelos investidores e dos termos da aquisição, entre outros, como explica Kravet (2009). Para Elton, Gruber, Brown \& Goetzmann (2014), os estudos de eventos são capazes de determinar se há geração de retornos anormais após a divulgação de informações relevantes. Devido à racionalidade e eficiência do mercado acionário, os efeitos de um evento, no caso o anúncio de uma Oferta Pública de Aquisição de Ações (OPA), são imediatamente traduzidos nos preços das ações da companhia realizadora da oferta, dessa forma, é possível medir o impacto de um determinado evento em uma companhia, pela variação anormal, por meio das cotações de suas ações comparadas aos índices de mercado, observam Gabrielli \& Saito (2003).

Esta pesquisa realiza estudos de eventos para verificar a possibilidade de evidências de retorno anormal no curto prazo, das empresas registradas na BMF\&Bovespa, no período de 2006 a 2015. As empresas estudadas foram as que adotaram política de recompra de ações através do método de OPA (fixed-price self-tender offer), no qual a empresa define o número de ações, a data de expiração e o pretenso preço da recompra.

A essência de um estudo de eventos se resume na tentativa de mensurar impactos nos preços de títulos em função de eventos ocorridos, colocam Campbell, Lo \& Mackinlay (1997). O pilar central é a hipótese de eficiência dos mercados, uma vez que os impactos de eventos relevantes seriam refletidos de maneira rápida no preço das ações. Essa metodologia consiste em sete passos conforme descritos na Figura 1:

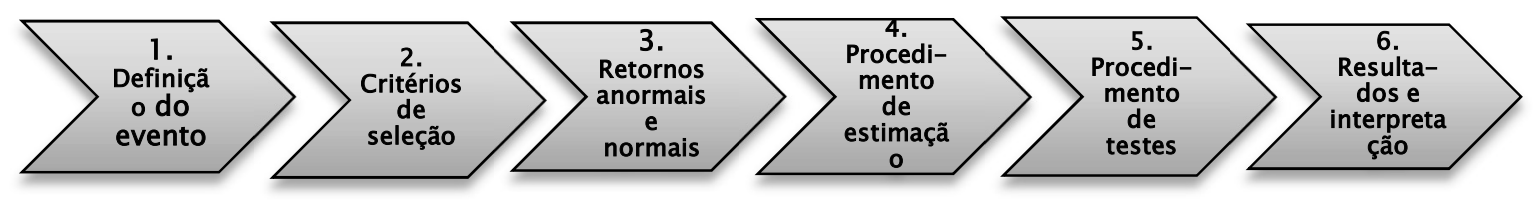

Figura 1 - Etapas de um estudo de evento. Fonte: Adaptado de Campbell, Lo \& Mackinlay (1997).

Este trabalho utiliza dados secundários extraídos da BM\&FBovespa no período de 2006 a 2015. Para operacionalização dos testes estatísticos utilizou o SPSS (Statistical Package for the Social Sciences). Com base no período estudado foram analisados 43 processos de OPA, em 41 empresas, conforme se apresenta a seguir. 


\subsection{DEFINIÇÃO DO EVENTO E JANELA DE EVENTO}

O evento analisado foi o processo de OPA realizado pelas empresas da amostra selecionada, no qual a respectiva data de ocorrência (data zero) foi definida como o dia de aprovação do processo de OPA, ou seja, a data da divulgação do fato relevante. Para a observação do fato, a janela de evento utilizada é de 21 dias antes da data zero, e 21 dias após o evento, incluindo o dia do evento (data zero), perfazendo um período de estudo total de 42 dias. Este intervalo foi escolhido, para assegurar a observação correta dos retornos anormais, uma vez que os preços dos ativos podem ser influenciados pelas notícias dos processos de oferta pública de ações.

\subsection{CRITÉRIOS DE SELEÇÃO DA AMOSTRA}

As amostras foram selecionadas através de fonte fornecida pela Economatica (2016), confirmada pelos dados coletados em BMF\&Bovespa (2016). Os eventos de OPA foram obtidos em pesquisa bibliográfica nos editais disponibilizados pela CVM (2016). Inicialmente, foram selecionadas todas as empresas que constavam em CVM (2016) com processos de OPA em um período de 10 anos, compreendido por 01/01/2006 e 31/12/2015. Foram elencados 65 processos de OPA em 59 empresas de capital aberto brasileiras, com periodicidade dos dados diária. Após a triagem, 43 processos de OPA foram analisados no teste de evento. O processo de seleção das amostras se deu devido à i) falta de liquidez diária das ações, ii) processos de OPA da mesma empresa em períodos inferiores a 90 dias, e iii) a falta de distribuição normal nas amostras.

A utilização de mais de um tipo de ação para a mesma companhia prejudica a independência dos eventos, na medida em que os retornos destas ações tendem a ser correlacionados. Para evitar este viés deve se escolher um único ativo, o que obtiver maiores retornos, explana Mackley (1997). No presente caso não foram excluídos processos de OPA somente por este critério, portanto não consta do rol de seleção.

O primeiro aspecto avaliado foi a liquidez diária destas ações. As empresas que apresentaram pregões consecutivos sem negociação dentro de sua janela de estimação ou de sua janela do evento foram excluídas da amostra, pois poderiam enviesar os eventos e, por conseguinte, os resultados. 
Pelo segundo aspecto excluíram-se dos ativos, as empresas que passaram por mais de um processo de OPA em períodos inferiores a 90 dias. Os processos de OPA foram assim escolhidos, pois conforme Mackley (1997) não estariam enviesados com eventos dos processos anteriores.

Um terceiro aspecto observado na exclusão de processos foi a não normalidade na distribuição das amostras. O Quadro 2 resume o critério de seleção das amostras, apresentando cada etapa do procedimento de escolha.

\begin{tabular}{|c|c|c|c|c|}
\hline Item & Item & Critério de exclusão & $\mathbf{N}^{0}$ OPAs & $\begin{array}{c}\mathrm{N}^{\mathrm{o}} \\
\text { Empresas }\end{array}$ \\
\hline & Inicial & Total de OPA entre 2006 e 2015 & 65 & 59 \\
\hline $\mathbf{I}$ & $1^{\mathrm{a}}$ exclusão & Falta de liquidez diária das ações & 12 & 12 \\
\hline Ii & $2^{\mathrm{a}}$ exclusão & $\begin{array}{c}\text { Processos em períodos inferiores a } 90 \\
\text { dias }\end{array}$ & 1 & 1 \\
\hline \multirow[t]{2}{*}{ Iii } & $3^{\mathrm{a}}$ exclusão & $\begin{array}{l}\text { Não normalidade na distribuição das } \\
\text { amostras }\end{array}$ & 9 & 5 \\
\hline & Final & OPAs selecionadas & 43 & 41 \\
\hline
\end{tabular}

Quadro 2 - Critério de seleção das amostras. Fonte: Elaborado pelos autores.

\subsection{RETORNOS NORMAIS E ANORMAIS}

Para os retornos normais foi admitida a hipótese do mercado perfeito, o que nos permite assumir que a média dos retornos simples do período da janela de estimação tende a se repetir em períodos futuros na ausência de informações relevantes, fornecendo então uma expectativa de retorno caso o evento não ocorresse, dessa forma, é esperado que a média dos retornos simples do período da janela de estimação tenderá a se repetir no período da janela de evento. O retorno anormal é obtido com a média dos retornos observados dentro da janela de evento menos a média dos retornos observados na janela de estimação. O evento de OPA é uma variável exógena, ou seja, é ela quem determinará uma alteração no retorno das ações, e não as ações vão acionar o evento. 


\subsection{PROCEDIMENTO DE ESTIMAÇÃO}

Para o procedimento de estimação foi considerada uma janela de estimação de 21 dias anterior à janela de eventos. Para a janela de comparação, foram considerados 21 dias após a janela de eventos, incluindo o dia do Fato Relevante (FR). O período total está descrito na Figura 2:

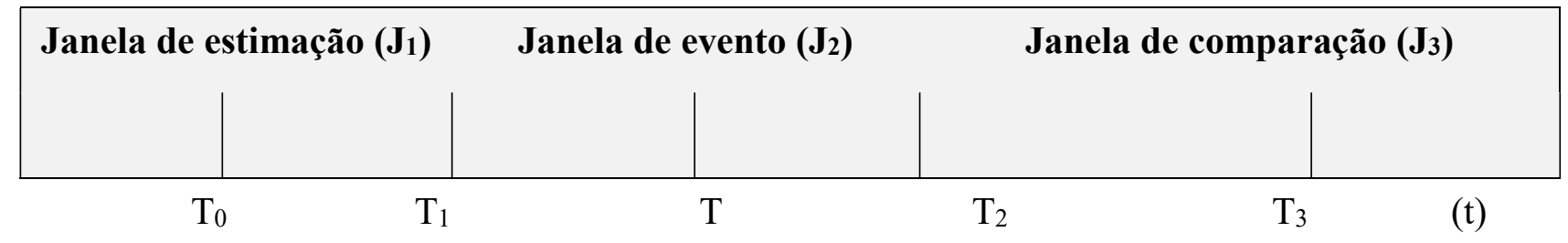

Figura 2 - Linha de tempo de um estudo de eventos. Fonte: Adaptado de Campbell, Lo \& Mackinlay (1997).

Sendo neste trabalho:

\begin{tabular}{|c|c|c|}
\hline Janelas & Período & Dias \\
\hline $\begin{array}{c}\text { Fato Relevante (data do } \\
\text { evento) }\end{array}$ & $\mathrm{T}$ & 1 \\
\hline Janela de estimação $\left(\mathrm{J}_{1}\right)$ & $\mathrm{J} 1=\mathrm{T} 1-\mathrm{T} 0$ & 21 \\
\hline Janela de evento $\left(\mathrm{J}_{2}\right)$ & $\mathrm{J} 2=\mathrm{T} 2-\mathrm{T} 1=\mathrm{T}$ & 20 \\
\hline Janela de comparação $\left(\mathrm{J}_{3}\right)$ & $\mathrm{J} 3=\mathrm{T} 3-\mathrm{T} 2$ & 1 \\
\hline
\end{tabular}

Quadro 3 - Janelas de estimação. Fonte: Elaborado pelos autores.

Assim, o período total de estudo é de 42 dias de negociação, dos quais, 21 ex ante e 21 ex post incluindo a data do evento ' $\mathrm{T}$ '. As janelas de estimação e de evento não são sobrepostas porque, segundo Campbell, Lo \& Mackinlay (1997) se houvesse a sobreposição, o evento poderia alterar os retornos normais enviesando os resultados da pesquisa. $\mathrm{O}$ dia do Fato Relevante (FR) adotado como a data do evento ' $\mathrm{T}$ ' foi incluso no período da Janela de Comparação, pois pela teoria da Eficiência do Mercado, a publicação dos FRs são imediatamente absorvidas pelo mercado e incorporada ao preço das ações. 


\subsection{PROCEDIMENTO DE TESTE}

No estudo de eventos, analisa-se o impacto de um evento mediante a subtração entre o retorno real verificado e retorno estimado em um curto período de tempo, chamado de retorno normal. Como o foco da pesquisa está em analisar o retorno anormal dos títulos $\left(\mathrm{AR}_{\mathrm{t}}\right)$ em relação a determinado evento - neste caso, a divulgação do evento de OPA - a equação (1) é utilizada para determinar os retornos anormais, esclarecem Campbell, Lo \& Mackinlay (1997).

$$
A R_{t}=R_{t}-E\left(\frac{R_{t}}{\beta_{t}}\right)
$$

Sendo:

1. $A R_{t}=$ o retorno anormal do ativo na data $\mathrm{t}$,

2. $R_{t}=$ o retorno real do ativo na data $\mathrm{t}$,

3. $E\left(\frac{R_{t}}{\beta_{t}}\right)=$ o retorno estimado do ativo na data $\mathrm{t}$, dado o retorno de $\beta_{t}{ }^{*}$.

Como neste trabalho o modelo de estimação escolhido foi o de mercado, $\beta_{t}$ corresponde ao Índice Bovespa (Ibovespa - definido como uma proxy do retorno de mercado).

O retorno real $R_{t}$ foi calculado utilizando a equação (2) de retornos ajustados ao mercado, que consiste em encontrar a diferença do retorno da ação em relação ao retorno do índice de mercado (Ibovespa) em uma mesma data, utilizando a forma logarítmica. De acordo com Favre \& Galeano, (2002) a função logarítmica obtém melhor aderência à distribuição normal dos retornos.

$$
R_{t}=\operatorname{Ln}\left(\frac{P_{t}}{P_{t-1}}\right)
$$

Os retornos diários do Ibovespa foram calculados de acordo com a Equação (3):

$$
R_{\text {ibov }}=\operatorname{Ln}\left(\frac{\text { Ibovespa }_{t}}{\text { Ibovespa }_{t-1}}\right)
$$

Sendo:

- $R_{\text {ibov }} \quad=$ retorno do Ibovespa no dia $\mathrm{t}$,

- Ibovespa $_{t}=$ a cotação média do Ibovespa no dia t, 
- Ibovespa $_{t-1}=$ a cotação média do Ibovespa no dia $\mathrm{t}-1$.

Como normalmente a janela de evento enquadra mais de um dia, para se interpretar os Retornos Anormais (AR) obtidos é necessário se estabelecer algum critério de agregação dos retornos anormais. A maneira mais comum é por meio da utilização do Cumulative Abnormal Return (CAR), que significa o retorno anormal acumulado explicam Campbell, Lo \& Mackinlay (1997). É obtido pela soma simples de todos os retornos anormais contidos em uma janela de evento:

$$
C A R_{i,(t 1, t 2)}=\sum A R_{t}
$$

Sendo:

- $C A R_{(t 1, t 2)}=$ retorno anormal acumulado do ativo $\mathrm{i}$

- $t_{1} \quad=$ primeiro dia da janela do evento.

- $t_{2} \quad=$ último dia da janela de evento.

O teste $t$ permite inferir sobre a igualdade de médias de duas amostras emparelhadas. Frequentemente cada caso é analisado duas vezes, antes e depois de um tratamento ou intervenção, formando pares de observações, cujas diferenças são testadas para ver se o resultado é ou não zero. O teste paramétrico tradicional, baseado na distribuição $t$-student, é obtido sob a hipótese de que a população tem distribuição normal. Nesse sentido, surge a necessidade de ser certificado se essa suposição pode ser assumida. Em alguns casos, assumir a normalidade dos dados é o primeiro passo que se toma para simplificar as análises. Para dar suporte a esta suposição, considerou-se, dentre outros, o teste de KolmogorovSmirnov (K-S). (Geweke, 1991, Kolmogorov \& Smirnov, 1967).

\section{RESULTADOS}

Os resultados e interpretação são apresentados a seguir, utilizando-se todos os eventos de OPA das empresas selecionadas.

Teste K-S para avaliar as hipóteses: 
$\left\{\begin{array}{l}\mathrm{H}_{0}: \text { Os dados apresentam uma distribuição normal } \\ \mathrm{H}_{1}: \text { Os dados não apresentam uma distribuição normal }\end{array}\right.$

Este teste observa a máxima diferença absoluta entre a função de distribuição acumulada assumida para os dados, no caso a normal, e a função de distribuição empírica dos dados. Como critério, compara-se esta diferença com um valor crítico, para um dado nível de significância. Dos 52 processos de OPA de 46 empresas, 9 processos de OPA (17\%) foram eliminados ( $p$-value $<0,05, \mathrm{H}_{0}$ rejeitada) por não apresentarem evidências de normalidade pelo teste K-S ao nível de significância de 95\%. Portanto, 43 processos de OPA de 41 empresas apresentam indícios de normalidade e estão aptas a se submeterem ao teste paramétrico $t$, como instrumento para a verificação de diferenças significativas ou não, ao nível $\alpha=0,05$ dos Resultados Anormais Acumulados (CAR) encontrados.

Para a realização do teste de hipótese, sobre a reação do preço da ação frente ao processo de OPA das empresas, foi usado a VIVO3 como exemplo, sendo que o mesmo processo aplicado a todos os demais ativos. O Fato Relevante (FR) ocorreu no dia 28/07/2010. As janelas de estimação ocorreram 21 dias antes do FR e 21 dias após, incluindo o próprio dia do FR.

Para o cálculo do Beta $(\beta)$ foram utilizados dados de 90 dias anteriores ao FR. Optouse por um período mais longo para atenuar possíveis efeitos de contágios pela possibilidade futura de ocorrência de OPA. No caso da empresa VIVO3 os dados para efeito do cálculo do $\beta$ se deram no período de 24/03/2010 até a véspera do FR, ou seja, 27/07/2010.

A janela de estimação $\left(\mathrm{J}_{1}\right)$ ocorreu de 29/06/2010 a 25/08/2010 e a janela de comparação (J3) de 28/07/2010 a 25/08/2010, incluindo a janela do evento 28/07/2010 (J2). 
CAR

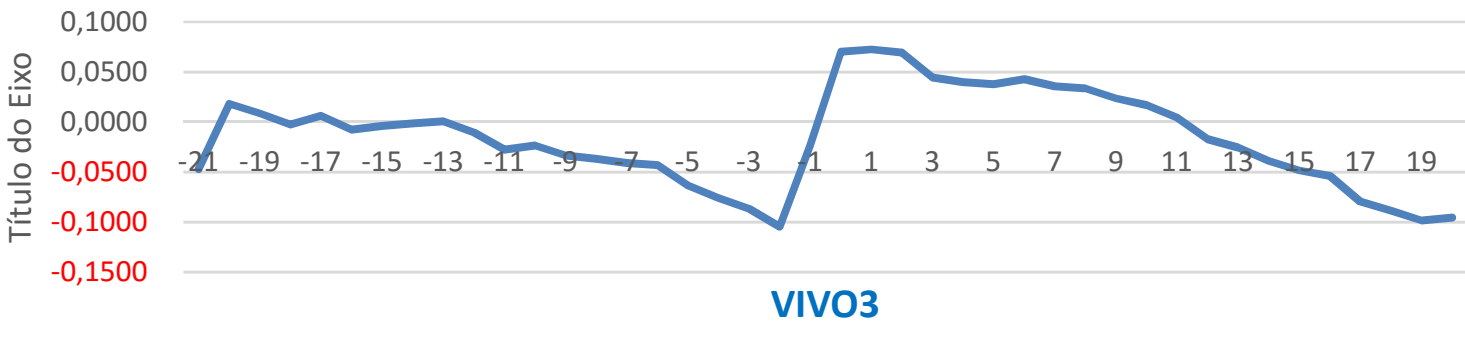

Figura 3 - Janelas de Eventos e CAR da VIVO3. Fonte: Elaborado pelos autores.

A Figura 3 apresenta as Janelas de Eventos e CAR da empresa VIVO3, utilizada como exemplo, sendo que o mesmo procedimento foi realizado para todos os 43 eventos de OPA.

Obtida a janela de estimação, foram calculados os coeficientes alfa e beta da regressão do retorno da ação das empresas, em relação ao retorno do mercado (IBovespa) por meio da utilização dos recursos Ferramenta de Análise do SPSS. Os retornos da ação e os retornos do mercado foram calculados utilizando-se a transformação logarítmica.

Os retornos esperados foram calculados com base no modelo de mercado, projetados por meio dos parâmetros alfa e beta descritos acima. Os Retornos Anormais (AR) foram obtidos como sendo a diferença entre retornos das ações e respectivos retornos esperados e, assim, o Retorno Anormal Acumulado (CAR) foi obtido pela soma dos AR calculados.

O teste $t$ exige que a distribuição seja normal. Fixam-se então as hipóteses. A hipótese nula $\left(\mathrm{H}_{0}\right)$ corrobora com o mercado não eficiente na forma semiforte, em que o preço não varia com o processo de OPA. A hipótese alternativa $\left(\mathrm{H}_{1}\right)$ corrobora com o mercado eficiente na forma semiforte, em que o preço varia com o processo de OPA.

$$
\begin{cases}\mathrm{H}_{0}: \quad \begin{array}{l}
\text { Preço da ação não varia com o processo de OPA, não há evidências de eficiência } \\
\text { do mercado na forma semi-forte. }
\end{array} \\
\mathrm{H}_{1}: \quad \begin{array}{l}
\text { Preço da ação varia com o processo de OPA, há evidências de eficiência do } \\
\text { mercado na forma semi-forte. }
\end{array}\end{cases}
$$

Para todas as empresas foi utilizada a mesma metodologia, ou seja, todas as janelas de estimação contêm 42 observações, e para todas as empresas, observa-se distribuição 
normal. Utiliza-se o teste $t$ das diferenças emparelhadas, para se comparar as CAR das duas Janelas de Eventos, ou seja, o período de 21 dias imediatamente anterior ao Fato Relevante (FR) com o período de 21 dias após o FR, incluindo o próprio dia do FR.

A Tabela 1 apresenta o resultado do teste $t$ para todos os 43 processos de OPA selecionados. A Figura 4 resume os resultados. Em 35 (81\%) o teste rejeita $\mathrm{H}_{0}$ (p-value < $0,05)$, indicando que em sua maioria os preços dos ativos variam com o processo de OPA, corroborando com a hipótese do mercado eficiente em sua forma semiforte. 


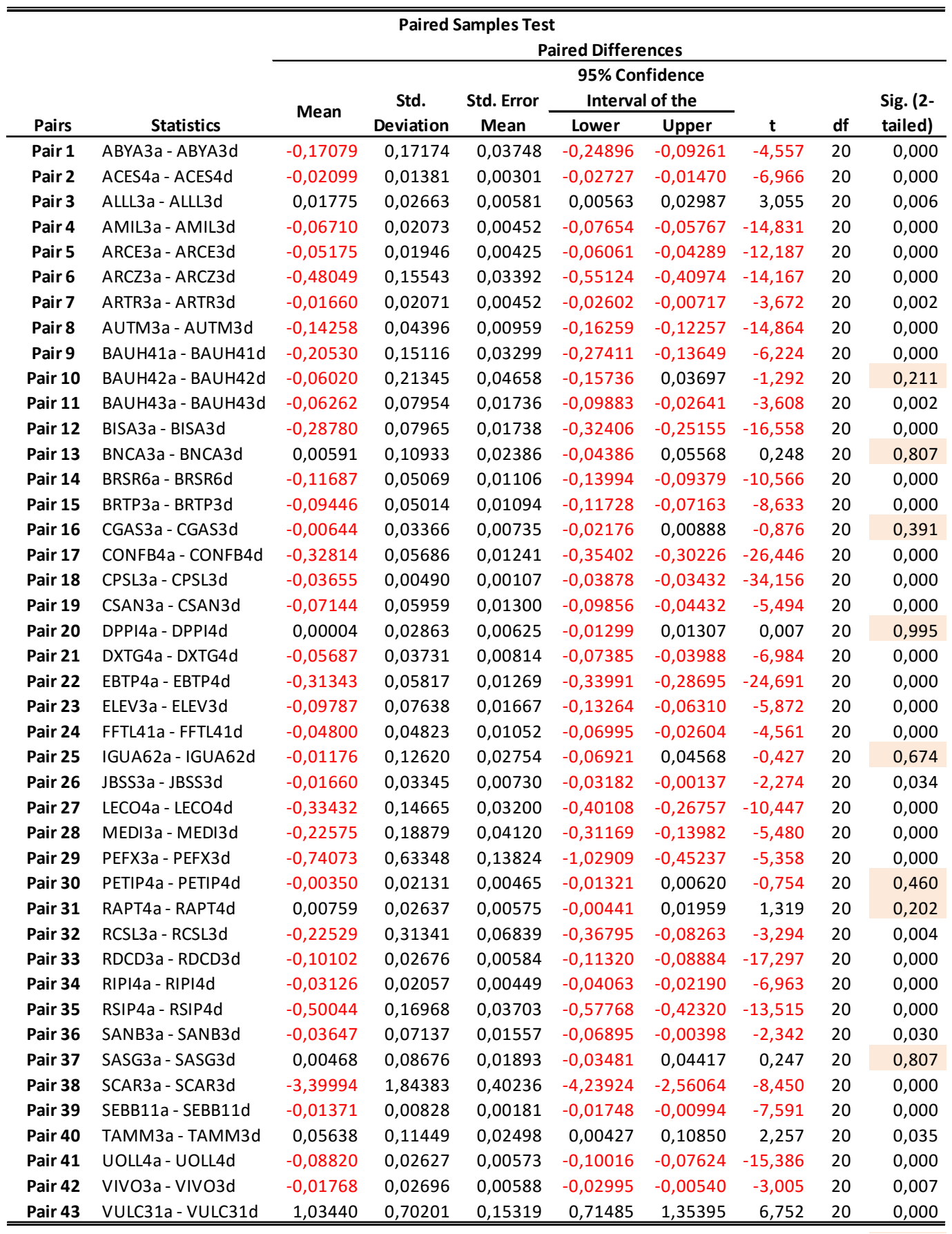

Tabela 1 - Teste $\boldsymbol{t}$ das diferenças emparelhadas. Fonte: Elaborado pelos autores. 


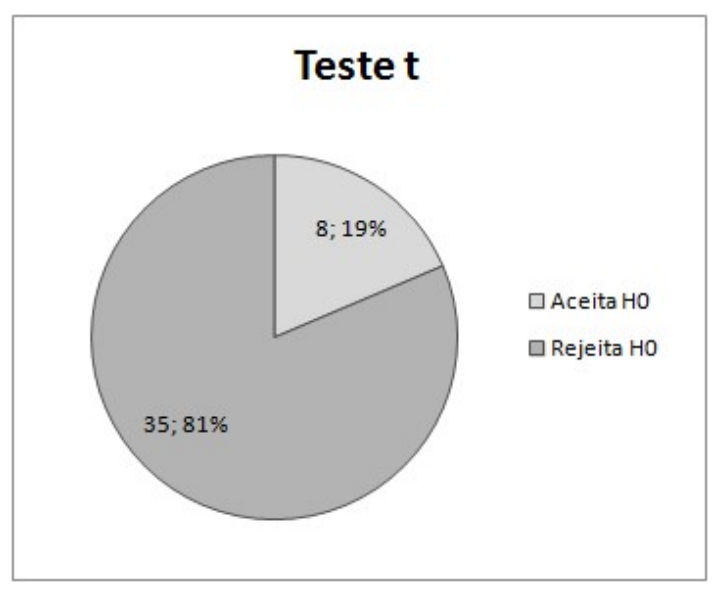

Figura 4 - Resultado do teste $t$ para os 43 processos.

Fonte: Elaborado pelos autores, 2017.

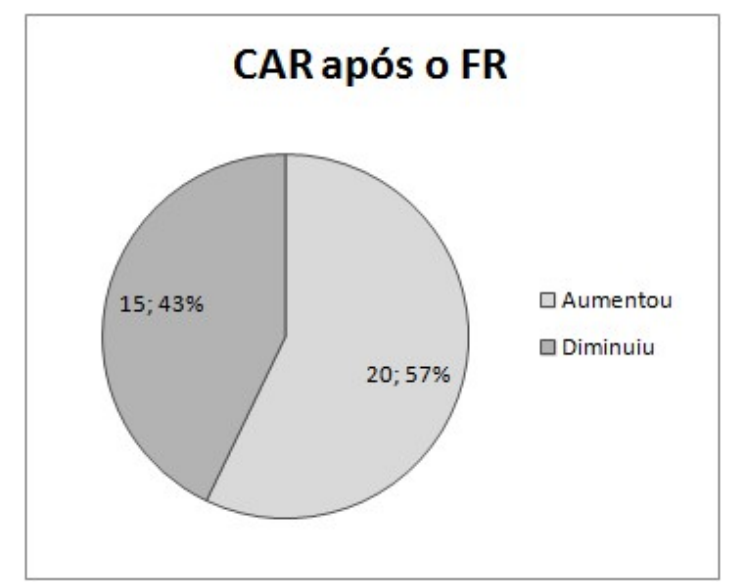

Figura 5 - Média das CAR após o FR em relação à média do período anterior. Fonte: Elaborado pelos autores, 2017.

Pela Figura 5 pode-se constatar que dos 35 processos de OPA com $\mathrm{H}_{0}$ rejeitadas, pouco mais da metade (57\%) teve a média da sua CAR aumentada depois do Fato Relevante (FR), quando comparada à média da sua CAR do período imediatamente anterior ao FR.

\section{CONSIDERAÇÕES FINAIS}

Esta pesquisa buscou verificar se os preços das ações sofreram variações em seus processos de operações públicas de aquisição de ações (OPA) no curto prazo. Foram selecionados para o estudo de Janelas de Eventos, 43 OPAs de 41 empresas, dentre 65 OPAs de 59 empresas em um período de 10 anos, compreendido entre 2006 e 2015. O processo de restrição na seleção das amostras se deu devido à i) falta de liquidez diária das ações, ii) processos de OPA da mesma empresa em períodos inferiores a 90 dias, e iii) a falta de distribuição normal nas amostras. O número de não normalidade na distribuição das amostras, 9 em 52 processos (17\%), se revelou significativo pelo teste de K-S, assim como a falta de liquidez diária das ações, 12 em 65 processos (18\%).

Este trabalho rejeitou a hipótese nula de que não haveria variação no preço das ações por conta do evento de OPA em 8 das 43 amostras selecionadas. Portanto, a maioria de 35 amostras (81\%) corrobora com a hipótese de mercado eficiente na forma semiforte, na janela de análise desde 21 dias anteriores ao Fato Relevante (FR) até 21 dias após, incluindo a data do FR. 
Em sua maioria, o resultado verificado no retorno acionário de curtíssimo prazo está consoante à hipótese de mercado eficiente na forma semiforte, uma vez que toda informação pública é absorvida pelos participantes do mercado de capitais e é imediatamente refletida no preço da ação.

Como sugestão para a continuidade da pesquisa, as janelas de eventos podem ser fragmentadas em 7, 14 e 21 dias, antes e após o FR, para verificação do comportamento das CAR em períodos menores. Um período mais longo, um ano ou mais poderia ser usado para a determinação dos regressores alfa e beta, a fim de amenizar o contágio pela possibilidade de evento de OPA futuro. Outra sugestão é reclassificar os resultados pelos objetivos das OPAs, como o fechamento de capital ou aquisição do controle de uma companhia aberta. Estudos podem ser elaborados explorando os efeitos das Instruções CVM n. 299 (1999), CVM n. 361 (2002), CVM n. 436 (2006), CVM n. 480 (2009) e CVM n. 487 (2010). Outro aspecto interessante a ser estudado é se há contágio no comportamento do retorno desses ativos em longo prazo, pelo evento das OPAs. Investigar e comparar o efeito de OPAs com o lançamento de novas ações em período longo, no mercado acionário brasileiro, como o deste estudo, também pode resultar em pesquisa relevante com contribuições à literatura pertinente.

\section{REFERÊNCIAS}

Berkovitch, E. \& Narayanna, M. P. (1993). Motives for takeovers: an empirical investigation. Journal of financial and quantitative analysis. 28(3), 347-36.

Brasil. (1999). Instrução CVM n. 299 de 9 de fevereiro de 1999. Procedimentos aplicados a OPA em companhias abertas. Acessado em 27 de fevereiro de 2016. Disponível em: http://www.cvm.gov.br/export/sites/cvm/legislacao/inst/anexos/200/inst299consolid.pdf.

Brasil. (2002). Instrução CVM n. 361 de 5 de fevereiro de 2002. Procedimentos aplicados a opa em companhias fechadas. Acessado em 27 de fevereiro de 2016. Disoponível em: http://www.cvm.gov.br/export/sites/cvm/legislacao/inst/anexos/300/inst361 consolidsem marcas.pdf.

Brasil. (2006). Instrução CVM n. 436 de 5 de julho de 2006. Anexo à instrução CVM $n$. 361 de 5 de fevereiro de 2002. Acessado em 27 de fevereiro de 2016. Disponível em: http://www.cvm.gov.br/legislacao/inst/inst361.html.

Brasil. (2009). Instrução CVM n. 480 de 7 de dezembro de 2009. Disposição sobre regimento de emissões de valores mobiliários admitidos a negociações e mercados regulados. Acessado em 27 de fevereiro de 2016. Disponível em: http://www.cvm.gov.br/legislacao/inst/inst480.html. 
Brasil. (2010). Instrução CVM n. 487 de 25 de novembro de 2010. Alteração da instrução CVM n. 361 de 5 de fevereiro de 2002. Acessado em 27 de fevereiro de 2016. Disponível em: de http://www.cvm.gov.br/legislacao/inst/inst480.html.

Brasil. (1976). Lei das sociedades por ações: Lei n. 6.404 de 15 de dezembro de 1976. Acessado em 27 de fevereiro de 2016. Disponível em: http://www.planalto.gov.br/ccivil_03/leis/L6404consol.htm

Brasil. (1976). Lei n. 6.385 de 7 de dezembro de 1976. Acessado em 27 de fevereiro de 2016. Disponível em: http://www.planalto.gov.br/ccivil_03/leis/L6385.htm

Bolsa de Mercadorias e Futuro e Bolsa de Valores de São Paulo - BMF\&Bovespa. Mercado de ações.(2016). Acessado em 01 de fevereiro de 2016. Disponível em: http://www.bmfbovespa.com.br/pt-br/mercados/acoes.aspx?idioma=pt-br.

Campbell, John. Y., Lo, Andrew. W. \& Mackinlay, A. Craig. (1997). The econometrics of financial markets. New jersey: Princenton University Prees.

Comissão de Valores Mobiliários - CVM (2016). Oferta pública de aquisição de ações (OPA). Recuperado em 01 de fevereiro de 2016, de http://sistemas.cvm.gov.br/?opa.

Comment, R. \& Jarrell, G. A. (1991). The relative signaling power of dutch-auction and fixed-price self-tender offers and open-market share repurchases. Journal of finance. .46, 243-1271.

Cremers, M., Nair V. B. \& John, K. (2009). Takeovers and the cross-section of returns. The review of financial studies. 22(4), 1409-1445.

Cziraki, Peter \& Michaely, Roni. (2016) What do insiders know? Evidence from insider trading around share repurchases and SEOs. Northern Finance Association Meetings in Mont Tremblant.

Dann, L. D. (1981). Commons stock repurchases: an analysis of returns to bondholders and stockholders. Journal of financial economics.9, 113-138.

Economatica (2016). Stocks. Recuperado em: ol de fevereiro de 2016, de: https://economatica.com/stocks.html. Acessado em 01/fev/2016.

Elton, E. J., Gruber, M. J., Brown, S. J. \& Goetzmann, W. N. (2014). Modern portfolio theory investment analysis. 9th edition. John Wiley \& Sons Ltd.

Etchebest, L. C., Costa, A.B., Silva, M. P. \& Pasqualeto, J. C. M. (2014). Recompra de ações: um estudo de eventos em períodos subsequentes. Anais do Congresso Nacional de Administração e Ciências Contábeis, Rio de Janeiro, RJ, Brasil, 5.

Evgeniou, T., Fortuny, E.J., Nassuphis, N. \& Vermaele, T. (2016). Share buyback and equity issue anomalies revisited. INSEAD Working Paper No. 2016/65/DSC/FIN.

Fama, E. F. (1970). Efficient capital markets. Journal of finance economics, 2, 383-417.

Fama, E. F. (1991). Efficient capital markets: ii. Journal of finance economics. 46, 15751617.

Favre, L. \& Galeano, J.A. (2002). Mean-modified value-at-risk optimization with hedge funds. The journal of alternative investments. 5, 21-25. 
FU, Fangjian \& HUANG, Sheng. (2015). The persistence of long-run abnormal returns following stock repurchases and offerings. Management Science. Research Collection Lee Kong Chian School of Business.

Gabrielli, M. F. \& Saito, R. (2003). Recompra de ações no Brasil: regulamentação e impactos sobre os minoritários. Anais do Encontro da associação nacional de programas de pós-graduação em administração - ANPAD, São Paulo, SP.

Geweke, J. (1991). Efficient simulation from the multivariate normal and student- $t$ distributions subject to linear constraints and the evaluation of constraint probabilities. Computing science and statistics: the twenty-third symposium on the interface. Seatle, Whashington, 23.

Gordon, A. (2001) Recompra de Ações: Uma abordagem Empírica. Dissertação de Mestrado em Economia da Escola de Pós-Graduação em Economia Fundação Getúlio Vargas. São Paulo.

Ikenberry, D., lakonishok, J. \& Vermaelen, T. (1995). Market underreaction to open market share repurchases. Journal of finance. 39, 181-20.

Jagannathan, M. \& Stephens, C. (2003). Motives for multiple open-market repurchase programs. Financial management. 32 (2), 716-791.

Jensen, M. C. (1986). Agency costs of free cash flow, corporate finance and takeover. American economic review. 76 (2), 323-329.

Kang, Jun-Koo; Kim, Jin-Mo; Liu, Wei-Lin \& Sougho, Sangho Yi. (2006). Post-takeover restructuring and the sources of gains in foreign takeovers: evidence from u.s. targets. The journal of business. 79(5), 2503-2537.

Kolmogorov-Smirnov, D. A. T. (1967). Distributional Adequacy the KolmogorovSmirnov: Goodness-of-Fit Test. Test, p. 1-5.

Lakonishok, J. \& Vermaelen, T. (1990). Anomalous price behavior around repurchase tender offers. The journal of finance, 45(2), 455-477.

Lambrecht, B. \& Myers, S. C. (2007). Theory of takeovers and disinvestment. The journal of finance. $62(2), 809-845$.

Mackinlay, A. C. (1997). Event studies in economics and finance. Journal of Economic Literature, 35(1), 13-39.

Manconi, A., Peyer, U., Vermaelen, T. (2015). Buybacks around the world market timing, governance and regulation. INSEAD Working Paper.

Massey Jr., F.J. (1951). The Kolmogorov-Smirnov Test for Goodness of Fit. Journal of the American Statistical Association. 46(253), 68-78.

Modigliani F. \& Miller, M.E. (1958). The cost of capital, corporation finance and the theory of investment. American economic review. 48, 261-297.

Moreira, L. F. (200). A Recompra de Ações na BOVESPA. Dissertação de Mestrado apresentada ao Programa de Pós-Graduação em Administração da Universidade Federal do Rio Grande do Sul. Porto Alegre. 
Nossa, S. N., Lopes, A. B. \& Teixeira, A. (2010). A Recompra de ações e a análise fundamentalista: um estudo empírico na Bovespa no periodo de 1994 a 2006. BBR Brazilian Business Review v.7, n.1 Vitória-ES, jan-abr 2010 p. 1-23 ISSN 1807-734X.

Peyer U. \& Vermaelen, T. (2009). The Nature and Persistence of Buyback Anomalies. INSEAD, Rev. Finance Study doi: 10.1093/rfs/hhn024.

Rogers, P. \& Mamede, S.P.N. (2015). Informational inefficiency and/or the value of liquidity? A study on the influence of market makers in brazil. Business and Management Review. ISSN: 2047-0398. ISSUE-V|4|N|5|, January|2015.

Ravenscraft, J. R. \& Scherer, F. (1987). Life after takeover. The journal of industrial economics. 36 (2), 147-156.

Ross, S. (1977). The determination of financial structure: the incentive signaling approach. Journal of economics. 8 (1). 23-40.

Vermaelen, T. (1981). Common stock repurchases and market signaling: an empirical study. Journal of Financial Economics, v.9, p. 139-83. 